EPJ Web of Conferences 106, 01006 (2016)

DOI: $10.1051 /$ epjconf/201610601006

(C) Owned by the authors, published by EDP Sciences, 2016

\title{
A Re-Evaluation of the Reference Environment at the WSMR Fast Burst Reactor
}

\author{
Mary Helen Sparks ${ }^{\mathrm{a}}$, and T. Michael Flanders \\ Reactor Physics Branch, SVAD, White Sands Missile Range, White Sands, NM, USA
}

\begin{abstract}
This paper reviews the primary reference field at $60.96 \mathrm{~cm}$ from core centreline and re-examines the reference environment with improved characterizations of the reactor structure, test fixtures and also addresses some smaller issues of non-tracking calculations by monte carlo methods with various cross section set generations. We are also addressing long standing issues of absolute normalization of integral fluence based on the original characterizations of the facility diagnostic instrumentation.
\end{abstract}

\section{Introduction}

It is important that the reactor community be able to accurately describe the radiation environment delivered to users of the facility. The original characterization of the MoLLY-G fast burst reactor was performed by Edgerton, Germeshausen \& Grier (EG\&G) [1] in the early 1960s. During the mid-1970s, users of the fast burst test reactors in the USA noticed inconsistent results during tests involving multiple facilities. Efforts to understand the role of dosimetry included simultaneous neutron spectrum and transistor damage measurement in a variety of test environments [2]. In the mid-1980s a renewed effort to improve the radiation environment characterization and standardization of test methods between the three US test reactor dosimetry laboratories [3] was begun.

While the effort to establish continuity between test facilities was under way, the parameters defining the environment were being updated. During the past thirty years, the silicon displacement damage/1 MeV equivalence, Shape Index and Hardness Parameter have changed several times as a result of improved nuclear data.

\section{Background}

The WSMR MoLLY-G reactor has a long history of use in many areas of nuclear research, development, testing, and evaluation. As an un-moderated, un-reflected, bare-critical assembly, it provides a slightly degraded fission spectrum. The assembly is operated in a 15.2 by 15.2 by 6.1 meter exposure cell constructed of thick concrete walls lined by gypsum and borated gypsum wall-board. The wall, floor, and ceiling neutron return component adds a 1/E tail to the primary fission leakage component.

\footnotetext{
${ }^{a}$ Corresponding author: mary.h.sparks4.civ@mail.mil
}

This is an Open Access article distributed under the terms of the Creative Commons Attribution License 2.0, which permits unrestricted use, distribution, and reproduction in any medium, provided the original work is properly cited. 


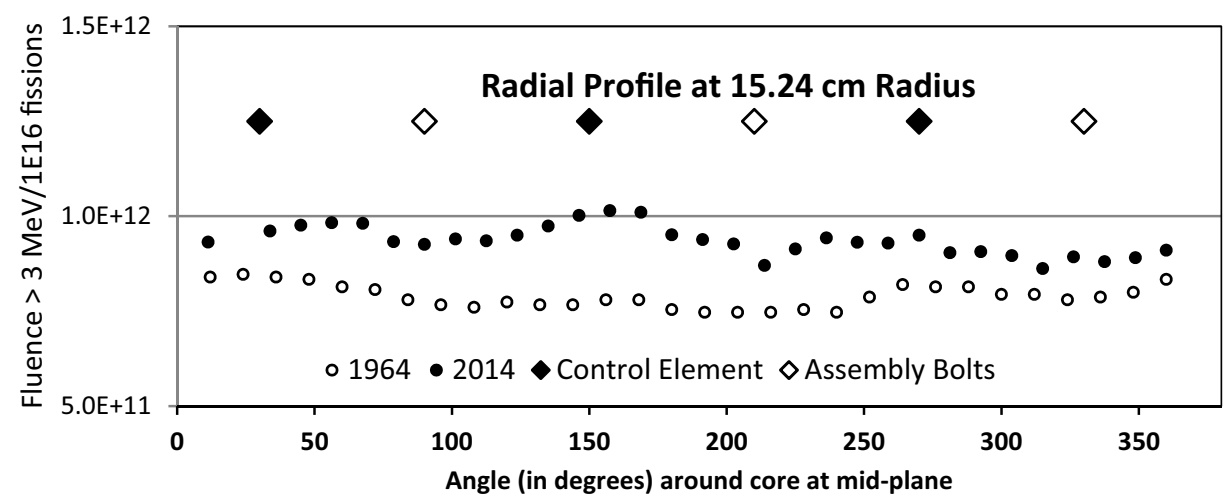

Figure 1. Horizontal fluence profile of MoLLY-G at mid-plane and $15.24 \mathrm{~cm}$ radius from core centreline.

The primary reference spectrum for the facility has been the position $60.96 \mathrm{~cm}$ from the core centerline. This location has been documented since 1976 [4], and has been extensively re-evaluated through the years $[5,6]$, both experimentally and by calculation. It serves as the basis for comparison to the exposure environments of other facilities and when used as the reference spectrum in national standards. This location is an optimal blend of distance from the source reducing the errors of experimental location for larger or unwieldy test items while maintaining the clean neutron spectrum with a well defined wall-return element. This location is particularly well suited to the primary mission of the facility, which is the exposure of electronic systems to intense neutron fields. Recently, use of the $15.24 \mathrm{~cm}$ location has increased significantly for testing. This is a report on the first steps to re-evaluate the reference test location for the facility and expand the scope of programs that can be supported with an increased confidence for additional locations equally well characterized.

\section{Facility Characterizations}

\subsection{Sulfur Metrics}

The original characterization data was taken in 1964 by EG\&G. Calibration of the sulphur activation monitors at that time was made by exposing the sulfur pellets $\left({ }^{32} \mathrm{~S}(\mathrm{n}, \mathrm{p})^{32} \mathrm{P}\right)$ to $14 \mathrm{MeV}$ neutrons from deuterium-tritium accelerators. Since 1980 the counting system has been calibrated by direct flux transfer methods using the National Institute of Standards and Technology, NIST, ${ }^{252} \mathrm{Cf}$ reference fields [7]. Figure 1 is a plot of the sulfur data from EG\&G in 1964 compared to 2014 data. For the original characterization, EG\&G placed 30 sulfur pellets on an aluminium ring with a $15.24 \mathrm{~cm}$ radius from core center-line and at mid-plane to the core. The 2014 exposure consists of 32 sulfur pellets surrounding the core also at $15.24 \mathrm{~cm}$. The 2014 pellets have been aligned to match the 1964 positions as closely as possible as shown in Fig. 1 . The data is reported as $\Phi>3 \mathrm{MeV} / 1 \times 10^{16}$ fission. The radial profile clearly shows the influence of the inconel bolts versus fuel control elements.

In addition to the horizontal exposure of sulfur pellets surrounding the machine for a radial representation, we also exposed in a vertical configuration. The vertical exposure, Fig. 2, was made with two stands placed $17.8 \mathrm{~cm}$ from core center and 180 degrees apart. The locations placed the stands adjacent to one control rod and the opposite stand was adjacent to a bolt. Each stand held eleven sulfur pellets with the center of the first pellet $1.27 \mathrm{~cm}$ above the test table. The pellets were placed on $2.54 \mathrm{~cm}$ centers to $27.94 \mathrm{~cm}$ high, the top of the safety shroud. The fluence maximum demonstrates the significance of placement accuracy at close-in positions. 


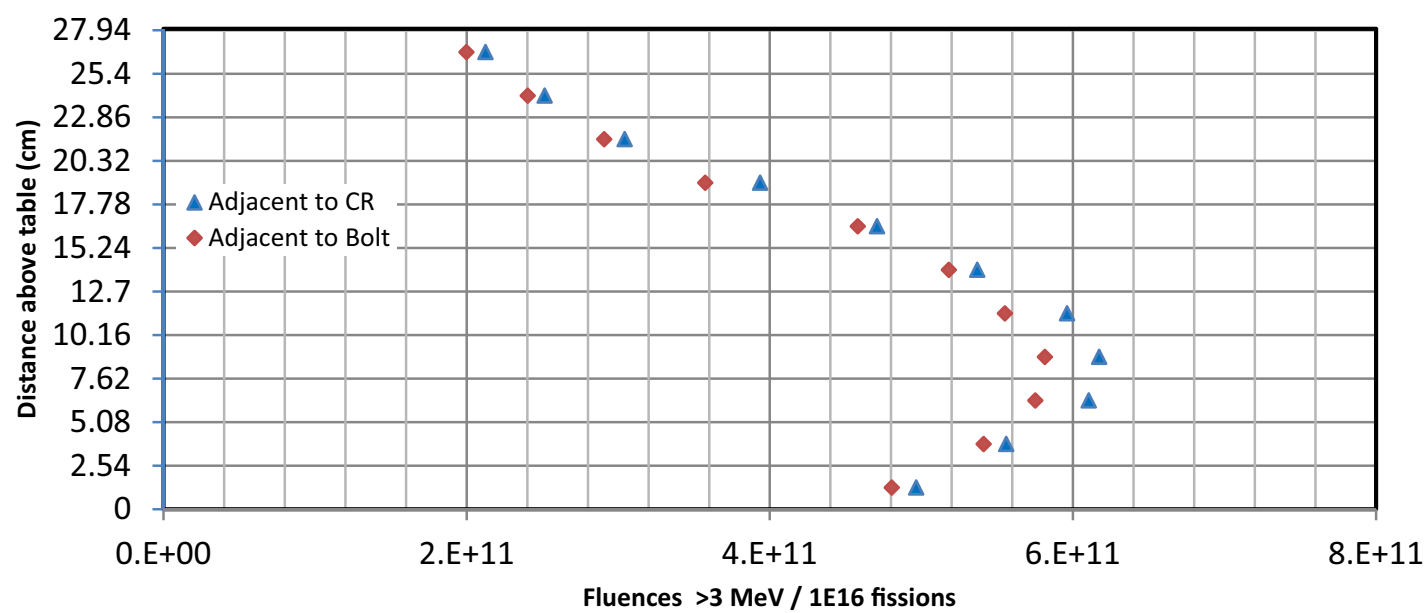

Figure 2. Vertical profile of fluences at the Molly-G at $17.78 \mathrm{~cm}$ from core centerline.

\subsection{Fission Yield Normalization}

Throughout the history of operations of the MoLLY-G, there has always been the requirement to provide a consistently reproducible radiation environment for life-cycle tests which may be performed over several years and for comparison with similar facilities. The simplest measure, from an operations perspective, is to record the power levels and integrated power during steady state operations and some measure of peak power in pulsed operations.

For the steady state mode of operations, the reactor power is monitored by a pair of power channels mounted on the exposure cell wall, approximately 7.6 meters away from the core. There are a number of factors that could interfere with consistent measurements made by compensated ion chambers. These include calibration and adjustment of the chambers inside their polyethylene shield and large experiments modifying the overall radiation environment in the cell, causing the detector to incorrectly interpret scattered radiation as direct radiation. Large experiments surrounding the core could also shield the detectors from measuring some fraction of the leakage radiation. For pulsed operations, the output of the reactor is proportional to the temperature rise in the core and can be measured by thermocouples. The thermocouples are not affected by changing external environments as are the power channels, but can provide suspect data as the contact between the thermocouple housing and the fuel can vary as the core suffers thermal shock during a series of pulses.

Reactor surveillance, maintenance, and calibration procedures minimize errors in reactor diagnostics to a great degree, but there have been a number of determinations of yield per fission and yield per observable diagnostic signal through the years. This resulted in differences in the measured fluence compared to reactor diagnostic. Calibration of dosimetry detector systems has undergone improvement through the years and has also contributed to the question of output of the reactor versus yield as measured by facility diagnostics.

The initial characterization of the radiation environment occurred immediately after the reactor was put into service in 1964. EG\&G published a comprehensive report detailing careful measurement of the neutron environment, reactor performance, and health physics measurements throughout the facility. Included as part of those measurements were fission yield determinations in both steady state and pulsed mode. EG\&G used 1.45 leakage neutrons/fission and $190 \mathrm{MeV}$ per fission to determine the integrated power output resulting value of $5.07 \mathrm{~kW}$-min per $1.0 \times 10^{16}$ fissions in a steady state operation. For pulsed operations, they determined a burst corresponding to $1.0 \times 10^{16}$ fissions resulted in a temperature 


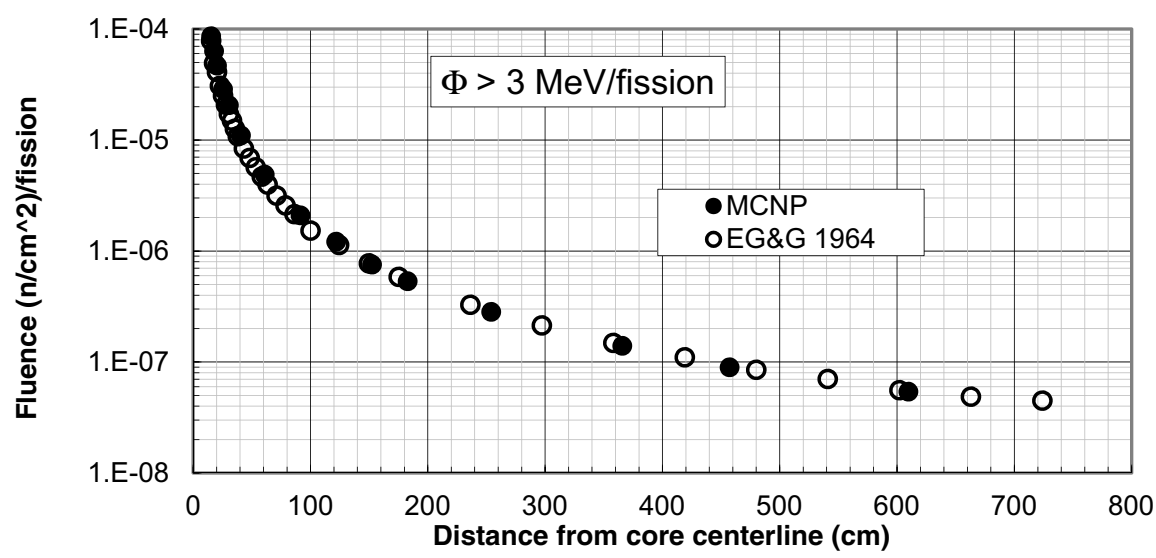

Figure 3. Fluence $>3 \mathrm{MeV}$ per fission as a function of distance from the core centerline.

Table 1. Fission Yield Normalization.

\begin{tabular}{|c|c|c|c|}
\hline & Pulse Operations & Steady State Operations & $\begin{array}{c}\text { Pulse/Steady State } \\
\text { (for Equivalent Yield) }\end{array}$ \\
\hline & ${ }^{\circ} \mathrm{C} / 1 \times 10^{16}$ fissions & $\mathrm{kW}-m i n / 1 \times 10^{16}$ fissions & ${ }^{\circ} \mathrm{C} / \mathrm{kW}-\mathrm{min}$ \\
\hline 1964 EG\&G & 24.3 & 5.07 & 4.79 \\
\hline MCNP4C \& ENDF/B5 & 27.7 & 6.86 & 4.04 \\
\hline MCNP5 \& ENDF/BVI & 27.4 & 6.78 & 4.044 \\
\hline
\end{tabular}

rise of $24.3{ }^{\circ} \mathrm{C}$, as measured by a reference thermocouple in the core. Their measurements were based on plutonium fission foils to determine fluence greater than $10 \mathrm{keV}$ and sulfur activation monitors to determine fluence greater than $3 \mathrm{MeV}$.

Our MCNP [8,9] calculations normalize data per fission neutron in k-code mode or per source neutron in fixed source mode. Using the original normalization constants determined by EG\&G has always resulted in some degree of offset in measured versus calculated spectra and fluence as measured by some activation foil monitors. Some discrepancy is due to the difference in sulfur calibration method. However, data collected since 1980 have shown that the reactor diagnostic providing power level vs. yield has remained very constant. The current experiments used sulfur activation monitors which are counted on a gas flow proportional counter. Since 1980, the yield per kW-min has stayed constant within about $2 \%$, including all power level calibrations, gas flow proportional calibrations, and experimental errors.

Figure 3 directly compares an early MCNP calculation with the original data collected by EG\&G using the original yield normalization factor. The agreement was very good with MCNP, only slightly over-predicting the fluence per fission. However, when compared to contemporary steady state measurement, the MCNP calculation will over-predict $\Phi>3 \mathrm{MeV}$ by more than $20 \%$.

Using the original $E G \& G$ data for equivalent $1.0 \times 10^{16}$ fission operations, the ratio of temperature rise in the core during a pulse to the steady state power level gives $4.79{ }^{\circ} \mathrm{C} / \mathrm{kW}$-min. For contemporary measurements $(\Phi>3 \mathrm{MeV}$ per degree temp. rise and $\Phi>3 \mathrm{MeV}$ per measured $\mathrm{kW}$-min), that ratio is $4.044^{\circ} \mathrm{C} / \mathrm{kW}$-min, a change of about $15 \%$. Based on the best available measurements, and detailed MCNP calculations for the experimental conditions with updated ENDF/B [10] cross sections, we have estimated a more appropriate set of yield normalizations listed in Table 1. The fission normalization constants in Table 1 are derived by normalizing calculated fluence with experimentally determined temperature rises and integrated power indicated by facility instrumentation. 


\section{$15^{\text {th }}$ ISRD}

\subsection{Shape Index \& Hardness Parameter}

An important parameter that provides a means of comparison of relative spectral shapes is the spectral index or the shape index. Spectral index is often used when comparing ratios of spectrum average cross sections for various facilities or sources. As a measurable quantity, it has the advantage of being directly measured and is simply a ratio of integral detector responses. The spectral index is widely used for inter-comparison environments of various facilities and comparisons to benchmark fields [7].

For tests involving the effects of neutron radiation on silicon devices, a more convenient set of comparison metrics have evolved through the years $[3,11,12]$.

$$
\begin{gathered}
\text { Shape Index }=\frac{\Phi_{\text {total }}}{\Phi>3 \mathrm{MeV}} \\
\text { Hardness Parameter }=\frac{\int_{0}^{\infty} \Phi(E) F_{D, \text { mat }}(E) d E}{F_{D, \text { Eref,mat }} \int_{0}^{\infty} \Phi(E) d E} \\
\Phi_{\text {eq,Eref,mat }}=\frac{\int_{0}^{\infty} \Phi(E) F_{D, \text { mat }}(E) d E}{F_{D, \text { Eref,mat }}}
\end{gathered}
$$

$\Phi(\mathrm{E})=$ incident neutron fluence spectrum

$\mathrm{F}_{\mathrm{D} \text {,mat }}(\mathrm{E})=$ neutron displacement damage function for the irradiated material (displacement damage per unit fluence) as a function of energy

$\mathrm{F}_{\mathrm{D} \text {,Eref,mat }}=$ displacement damage reference value designated for the irradiated material and for the specified energy, Eref.

For routine exposures, the neutron fluence is measured by activating sulfur monitors which provide the neutron fluence greater than $3 \mathrm{MeV}$. The $1-\mathrm{MeV}$ equivalent fluence for silicon displacement damage is then found using the experimentally dependent shape index and hardness parameter. The original limits of integration were $10 \mathrm{keV}$ and $18 \mathrm{MeV}$ since most of the displacement damage in silicon devices occurs at energies of $10 \mathrm{keV}$ or greater and the original ASTM standard only provided displacement damage function data between $10 \mathrm{keV}$ and $18 \mathrm{MeV}$.

More recently, we have used Shape Index and re-defined the Hardness Parameter to include the damage caused by lower energy neutrons with the integration limits now below thermal neutron energy and extending to $20 \mathrm{MeV}$ for silicon. In practice, the integration limits can still be chosen as $\mathrm{E}_{\min }$ and $\mathrm{E}_{\mathrm{max}}$ providing they give the same result as if the integrations were from 0 to $\infty$.

Our assessment of the general shape of the neutron spectrum has changed slightly through the years because of increased accuracy in the activation foil measurements and the continuous updating of the cross section libraries $[13,14]$. The last major update to the shape of the damage function occurred in the mid-1980s with a rather significant change to the value of $F_{D \text {,Eref }}$ for silicon. The impact of change to the test communities can be significant. The value of $\mathrm{F}_{\mathrm{D}, 1-\mathrm{MeV}, \mathrm{Si}}$ during the lifetime of the MoLLY-G reactor has changed from $78 \mathrm{MeV}$-mb in $1978,84 \mathrm{MeV}$-mb in 1986, and is now $95 \mathrm{MeV}$-mb. This has been the primary reason the 1-MeV Equivalence of the test spectrum has changed from 1.12 to its current value of 0.867. Current reference data for selected test positions are provided in Table 2.

When reviewing older exposure data, it is important to take into account the changes resulting from altering the definition of the parameters which determine the $1-\mathrm{MeV}$ equivalence of the test exposure. Special care must be taken to account for changes for the $\mathrm{F}_{\mathrm{D}, 1-\mathrm{MeV}, \mathrm{Si}}$ reference value. 
Table 2. Spectral parameters for silicon displacement damage for the MoLLY-G Reactor.

\begin{tabular}{|c|c|c|c|c|c|c|}
\hline Distance from core centerline & \multicolumn{3}{|c|}{ Shape Index } & \multicolumn{3}{c|}{ Hardness Parameter } \\
\hline cm (in) & Calc & Meas & C/M & Calc & Meas & C/M \\
\hline $15.24(6)$ & 7.86 & 7.85 & 1.00 & 0.913 & 0.893 & 1.02 \\
\hline $25.4(10)$ & 7.68 & 7.85 & 0.978 & 0.907 & 0.893 & 1.02 \\
\hline $60.96(24)^{*}$ & 8.20 & 8.02 & 1.02 & 0.873 & 0.865 & 1.01 \\
\hline $121.9(48)$ & 9.14 & 8.63 & 1.06 & 0.812 & 0.817 & 0.994 \\
\hline $254 .(100)$ & 12.0 & 12.0 & 1.00 & 0.688 & 0.657 & 1.05 \\
\hline
\end{tabular}

* The $60.96 \mathrm{~cm}$ position is used as the baseline reference when comparing to other spectra and facilities.

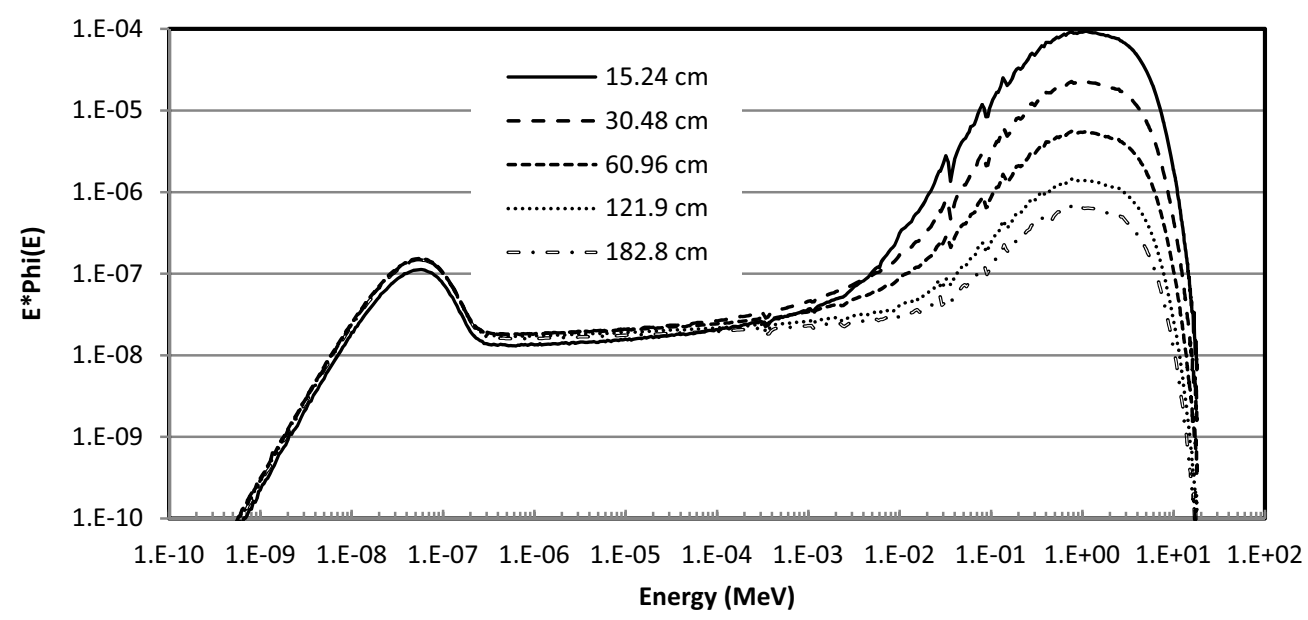

Figure 4. E*Phi(E): Spectra calculated at 5 distances from core centerline.

\section{Improvements to Predictive Modelling}

\subsection{MoLLY-G}

Various codes have been used for calculations for the MoLLY-G. The early 2-dimensional calculations were run with the Two-Tran discrete ordinates codes [15]. These calculations were used mostly during the design phase of the reactor core. Later calculations using DOT-III [15] improved the definition of the radiation environment external to the core and included a two-dimension representation of the reactor exposure cell [4]. These model improvements proved to be very useful in estimating the neutron spectra external to the core and the effects of the environment.

In the mid 1980s, MCNP became one of the standard codes for simulation modelling at WSMR. With increased computer capability, we improved the model of the MoLLY-G facility, Fig. 4. The exposure room configuration has remained constant through the years. We are currently updating the detail of the reactor core, the reactor support stand, and current experimental exposure table. Additional changes are in process for a new experimental table, experimental framework, and safety shroud. As these changes are finalized, we propose to perform detailed calculations and experiments of the new reference environments.

\subsection{Cross-Section Sets ENDF/B-V, B-VI, B-VII}

A simplified reactor core model has been used for evaluating the effect of changing cross section data. MCNP has been used since the 1980s with a variety of cross sections, beginning with ENDL [16], 


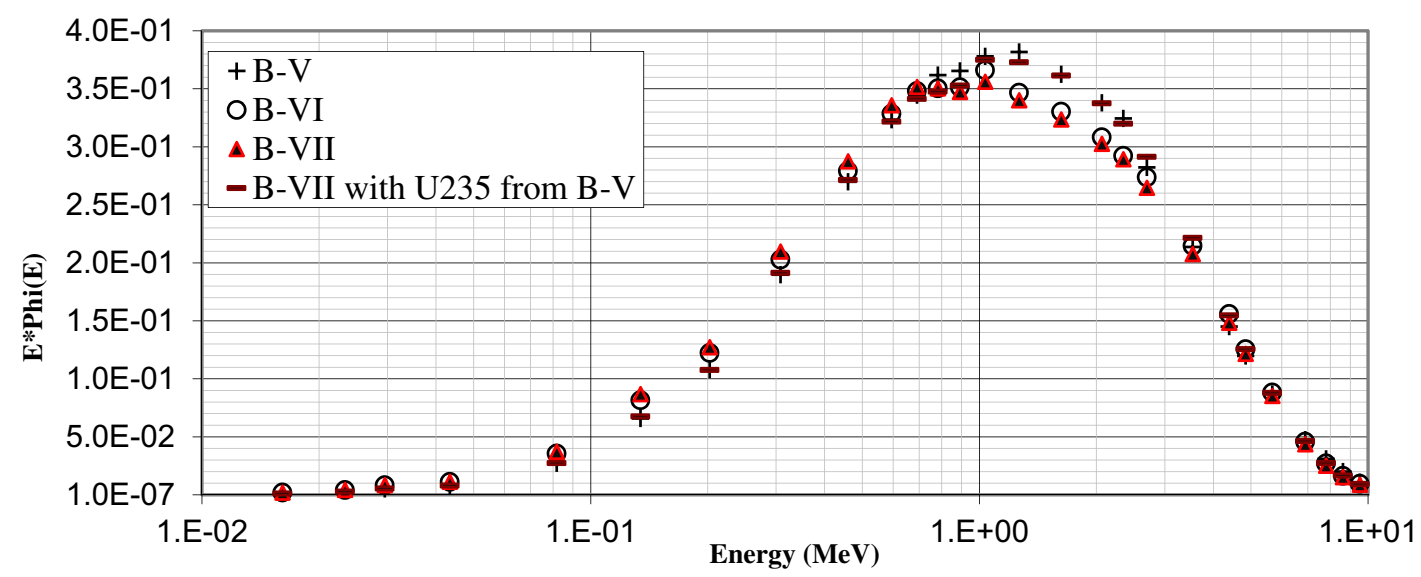

Figure 5. Spectra calculated with a simplified core model using various cross section sets: $60.96 \mathrm{~cm}$ location.

early ENDF/B libraries, and special Los Alamos evaluations. Through the history of calculations up through ENDF/B-V, the variations in our calculated spectra were almost unobservable. This is true both for calculations of the simple early models of the core through the more detailed models of the core in the exposure cell. However, beginning with ENDF/B-VI, small variations in the spectra were observed. Calculated $\mathrm{k}_{\text {eff }}$ values also did not track beginning with B-VI. The current version of the MCNP code is distributed with B-VII cross sections. Simple initial comparisons show the B-VII based calculations are tracking with the B-VI based calculations. Figure 5 shows a comparison of a region near the peak of the fission spectrum. The spectrum based on the B-V cross sections peaks about 5 to $10 \%$ higher than similar calculations with B-VI and B-VII cross sections. Additional runs with the B-VI and B-VII cross section sets, except for replacing the ${ }^{235} \mathrm{U}$ cross sections with the earlier B-V based cross sections, shows the change appears to be primarily attributable to the change in ${ }^{235} \mathrm{U}$ cross sections. Changing other individual cross sections, for example, ${ }^{238} \mathrm{U}$, did not prove to show the same effect. It is anticipated based on calculations to date, that as we update our reference spectra, the change in neutron spectra will result in changes to our response function conversion factors, with the changes being lower on the order of a few per cent. A complete set of new reference spectra folded with a variety of response functions, such as the 1-MeV Si displacement damage function will be examined in future work.

\section{Summary}

After many years of only minor changes to the evaluations of the radiation environment of the MoLLY-G reactor, changes in recommended cross section sets, facility configuration and improved capability for generating detailed models of the reactor and environment, there is a need to update our characterizations. We have compared new measurements of the neutron environment with the original data of EG\&G. We have re-evaluated the neutron yield versus reactor power level calibration and burst diagnostics.

We have begun a program to update our reference environment at $60.96 \mathrm{~cm}$ from core centerline. This will include more detailed modelling of the reactor, the support structure and experimental fixtures. In addition to these improved models, there will be a need to establish a better configuration control mechanism to ensure the consistency required for a reference environment. 


\section{References}

[1] K. Humpherys, W. Quam, C. Rainbolt, R. Durkee and T. Dahlstrom, "Nuclear Radiation Dosimetry Measurements of the White Sands Missile Range Fast Burst Reactor", Edgerton, Germeshausen \& Grier, Santa Barbara Division, Report S-262-R (1965)

[2] V. V. Verbinski, C. Cassapakis, R.L. Pease and H. L. Scott, "Simultaneous Neutron Spectrum and Transistor-Damage Measurements in Diverse Neutron Fields: Validity of $\mathrm{D}_{\mathrm{Si}_{\mathrm{i}}}\left(\mathrm{E}_{\mathrm{N}}\right)$ ", Report 3929, Naval Research Laboratory (1979)

[3] M.H. Sparks, T.M. Flanders, J.G. Williams, J.G. Kelly, W.W. Sallee, M. Roknizadeh and J.L. Meason, "Energy Dependence on Neutron Damage in Silicon Bi-Polar Transistors", IEEE Transactions on Nuclear Science, Vol. 35, No 6, pp 1904 (1989)

[4] J.L. Meason, H.L. Wright, J.C. Hogan, J.T. Harvey, "The Neutron Spectral Distribution from a Godiva-Type Critical Assembly," IEEE NS-22, No. 6 (1975)

[5] T.M. Flanders, M.H. Sparks, "Calculated Neutron Spectra of Fast Pulse Reactors," Proceedings of the Seventh ASTM-Euratom Symposium on Reactor Dosimetry, Strasbourg, France, Kluwer Academic Publishers (1990)

[6] T.M. Flanders, M.H. Sparks, W.W. Sallee, "Radiation Environment Produced by the Whites Sands Missile Range MoLLY-G Reactor," Proceedings of the Physics, Safety and Application of Pulse Reactors International Embedded Topical Meeting, ANS (1994)

[7] J.A. Grundl, C.M. Eisenhauer, "Compendium of Benchmark Neutron Fields for Reactor Dosimetry," NBSIR 85-3151, U.S. Department of Commerce (1986)

[8] X-5, Monte Carlo Team, "MCNP-A General Monte Carlo N-Particle Transport Code Version 5", report LA-CP-03-0245, Los Alamos National Laboratory, Los Alamos, NM (2003)

[9] T. Goorley et al, "Initial MCNP 6 Release Overview," LA-UR-11-07082, Los Alamos National Laboratory, also Nuclear Technology, 180, pg 298-315 (Dec. 2012)

[10] See Appendix G of most (Version 4 and later) MCNP manuals for a detailed discussion of cross section sets (ENDF/B/others) used in this code, Los Alamos National Laboratory, Los Alamos, $\mathrm{NM}$

[11] T.F. Luera, P.J. Griffin, J.G. Kelly, M.S. Lazo, "Simulation Fidelity in Reactor Irradiation of Electronics", Proceeding of the Seventh ASTM-EURATOM Symposium on Reactor Dosimetry, Kluwer Academic Publishers (1992)

[12] ASTM Standard Practice E722, "Standard Practice for Characterizing Neutron Fluence Spectra in Terms of an Equivalent Monoenergetic Neutron Fluence for Radiation Hardness Testing of Electronics," ASTM Standard Practice E722-09². American Society for Testing and Measurement, West Conshohocken, PA (2013)

[13] P.J. Griffin, J.G. Kelly, T.F. Luera, "SNL-RML, Recommended Dosimetry Cross section Compendium,, SAND92-0094, Sandia National Laboratories, Albuquerque, NM (1994)

[14] M.H. Sparks, W.W. Sallee, T.M. Flanders, "Investigation of Radiation Transport Modelling Trends in the WSMR Fast Burst Reactor Environments", Reactor Dosimetry: $12^{\text {th }}$ International Symposium, STP1490, Journal of ASTM International, pp 30-305 (2008)

[15] Radiation Safety Information Computational Center, Oak Ridge National Laboratory, Oak Ridge, TN

[16] R.J. Howerton, D.E. Cullen, R.C. Haight, M.H. MacGregor, S.T. Perkins and E.F. Plechaty, "The LLL Evaluated Nuclear Data Library (ENDL): Evaluation Techniques, Reaction Index, and Descriptions of Individual Reactions," Lawrence Livermore National Laboratory Report UCRL50400, Vol. 15, Part A (1975) 